Meta

Journal des traducteurs

Translators' Journal

\title{
La investigación sobre la traducción entre catalán y español: estudios traductológicos
}

\section{Cristina García de Toro}

Volume 60, numéro 3, décembre 2015

URI : https://id.erudit.org/iderudit/1036143ar

DOI : https://doi.org/10.7202/1036143ar

Aller au sommaire du numéro

\section{Éditeur(s)}

Les Presses de l’Université de Montréal

ISSN

0026-0452 (imprimé)

1492-1421 (numérique)

Découvrir la revue

Citer cet article

García de Toro, C. (2015). La investigación sobre la traducción entre catalán y español: estudios traductológicos. Meta, 60(3), 557-575.

https://doi.org/10.7202/1036143ar
Résumé de l'article

Cet article constitue la deuxième partie d'une vaste recherche consacrée aux travaux sur la traduction entre le catalan et l'espagnol, aussi bien ceux qui se fondent sur la linguistique que ceux qui se basent sur l'approche traductologique. La première : " La investigación sobre la traducción entre catalán y español : estudios lingüísticos », qui abordait la recherche faite dans le domaine de la linguistique, a été publié dans un numéro antérieur. La deuxième est cet article qui s'occupe de la recherche effectuée en traductologie. Le but du premier article était d'offrir une approximation de ce champ d'étude à partir d'une approche linguistique. Le but de ce deuxième article est de compléter la cartographie de la recherche en étudiant les travaux du domaine traductologique. Les travaux se fondant sur la traductologie sont centrés sur la description des caractéristiques spécifiques de la traduction entre le catalan et l'espagnol, tout en l'intégrant dans une théorie générale de la traduction. Bien que l'intérêt pour la traduction entre le catalan et l'espagnol soit relativement jeune, nous comptons avec des approches historiques et des approches fondées sur l'enseignement de cette paire de langues. Une troisième ligne de recherche est constituée par les réflexions que la pratique de la traduction suscite dans les milieux professionnels. Donc, la structure de l'article suit ces trois approches : études historiques, études descriptives des variétés de traduction et études appliquées. Nous établirons des dénominateurs communs entre les travaux étudiés qui nous aideront à définir les voies de recherche actuelles et à ébaucher les voies futures.
Tous droits réservés @ Les Presses de l’Université de Montréal, 2015
Ce document est protégé par la loi sur le droit d'auteur. L’utilisation des services d'Érudit (y compris la reproduction) est assujettie à sa politique d'utilisation que vous pouvez consulter en ligne.

https://apropos.erudit.org/fr/usagers/politique-dutilisation/ 


\title{
La investigación sobre la traducción entre catalán y español: estudios traductológicos
}

\author{
CRISTINA GARCÍA DE TORO \\ Universitat Jaume I, Castelló de la Plana, España \\ garciat@trad.uji.es
}

\section{RÉSUMÉ}

Cet article constitue la deuxième partie d'une vaste recherche consacrée aux travaux sur la traduction entre le catalan et l'espagnol, aussi bien ceux qui se fondent sur la linguistique que ceux qui se basent sur l'approche traductologique. La première: «La investigación sobre la traducción entre catalán y español: estudios lingüísticos», qui abordait la recherche faite dans le domaine de la linguistique, a été publié dans un numéro antérieur. La deuxième est cet article qui s'occupe de la recherche effectuée en traductologie. Le but du premier article était d'offrir une approximation de ce champ d'étude à partir d'une approche linguistique. Le but de ce deuxième article est de compléter la cartographie de la recherche en étudiant les travaux du domaine traductologique. Les travaux se fondant sur la traductologie sont centrés sur la description des caractéristiques spécifiques de la traduction entre le catalan et l'espagnol, tout en l'intégrant dans une théorie générale de la traduction. Bien que l'intérêt pour la traduction entre le catalan et l'espagnol soit relativement jeune, nous comptons avec des approches historiques et des approches fondées sur l'enseignement de cette paire de langues. Une troisième ligne de recherche est constituée par les réflexions que la pratique de la traduction suscite dans les milieux professionnels. Donc, la structure de l'article suit ces trois approches: études historiques, études descriptives des variétés de traduction et études appliquées. Nous établirons des dénominateurs communs entre les travaux étudiés qui nous aideront à définir les voies de recherche actuelles et à ébaucher les voies futures.

\section{ABSTRACT}

This article constitutes the second part of a wider research devoted to investigation carried out on translation between Catalan and Spanish, both from the fields of linguistics and translation theory. The first one, entitled "La investigación sobre la traducción entre catalán y español: estudios lingüísticos," which tackled research on Catalan-Spanish translation done in the field of Linguistics, has been recently published in a former issue. The second one is this article focused on research done on Catalan-Spanish translation in the field of Translation Studies. While the aim of the first article was to provide researchers with an initial approach to the field of study of Catalan-Spanish translation from some perspectives grounded in the field of Linguistics, the aim of this second article is to complete the whole map of research in this field with the lines of investigation developed in the discipline of Translation Studies. Research from translation studies essentially describes the specific features of Catalan-Spanish translation within a general theory of translation. Although the discipline of Translation Studies is still relatively young and so it is the study of some language pairs, we still can count on historical approaches on Catalan-Spanish translation and also on approaches based on the teaching of this language pair. A third line of research is the one constituted by the practice of translation in the professional circles. Therefore, the structure of the article follows these three approaches: historical studies, descriptive studies on translation varieties and domains and applied studies. The article also identifies common denominators in the pieces of research examined, which help define current lines of research and shape future ones. 


\section{MOTS-CLÉS/ KEYWORDS}

traduction catalan-espagnol, langues en contact, catalan, interférence, linguistique contrastive

translation Catalan-Spanish, contact languages, Catalan, interference, contrastive linguistics

Este artículo representa la continuación de un artículo precedente, titulado: «La investigación sobre la traducción entre catalán y español: estudios lingüísticos», aparecido en esta revista con anterioridad (Del Toro 2015). Completa por tanto un estudio amplio cuyo objetivo ha sido revisar los trabajos dedicados a la traducción entre el par de lenguas formado por el catalán y el español, tanto los que se han interesado por la dirección del español al catalán, como por la opuesta, del catalán al español. El estudio se ha realizado en dos fases, en la primera nos hemos ocupado de los trabajos dedicados a la traducción entre estas dos lenguas procedentes de una disciplina de larga tradición como es la lingüística, y, en esta segunda fase, hemos tratado los trabajos enmarcados en la traductología, aún escasos e incipientes.

En el marco de los estudios de traducción, la investigación dedicada específicamente a la traducción entre catalán y español es aún incipiente, a pesar de que los problemas derivados de los contactos entre lenguas han sido tema de interés constante para la traductología: Vinay y Darbelnet (1958) y Guillemin-Flescher (1981) se han ocupado de la combinación francés-inglés); Intravaia y Scavée (1979) del francés y el italiano; Malblanc (1961) del francés y el alemán; y Vázquez Ayora (1977) del inglés y el castellano. Sin embargo, la traducción entre catalán y español no ha despertado aún suficiente interés en la disciplina. Son muchos los ámbitos en los que no contamos con estudios o en los que el interés es tangencial por formar parte de trabajos más amplios. Por lo que investigar en este campo nos ha conducido irremediablemente a buscar referencias en disciplinas próximas, de más larga tradición en el estudio del contacto entre lenguas. Entre ellas, la lingüística es las que más directamente se ha interesado por el español y el catalán y su traducción, en especial la lingüística contrastiva, como mostrábamos en el artículo mencionado. La revisión de los trabajos procedentes de la lingüística nos permitió concluir que su interés residía fundamentalmente en los fenómenos derivados de la interferencia y sus implicaciones en la estandarización ortográfica y gramatical de ambas lenguas, en especial del catalán, durante el proceso de normalización de la lengua. La depuración de interferencias léxicas constituía el otro elemento nuclear.

La finalidad del presente artículo, que recoge ahora los trabajos enmarcados en la traductología, es ofrecer un primer acercamiento a este campo de estudio, con el objeto de contribuir a su visibilidad. Para ello, estableceremos unos denominadores comunes entre los trabajos encontrados, que definen las líneas de investigación actuales y contribuyen a perfilar las futuras, pero conscientes de que este es solo un primer esbozo, sin pretensión de exhaustividad y conscientes también de que las ausencias serán significativas. La aparición de la investigación sobre la traducción entre español y catalán está ligada al momento histórico y a los condicionantes sociogeográficos y políticos que gravitan en torno a esta práctica de traducción, por lo que ha sido necesario que el proceso de normalización del catalán se pusiera en marcha para hacer posibles unas condiciones favorables para la investigación. Por esta razón, 
todos los trabajos que hemos recogido en los siguientes apartados son muy recientes, son trabajos que comienzan a publicarse, con pocas excepciones, a partir de la década de los 90 .

A pesar de su juventud, sin embargo, se pueden trazar ya las líneas principales que va siguiendo la investigación sobre la traducción entre catalán y español: por una parte contamos con estudios interesados por su reconstrucción histórica, por otra, con las reflexiones que la docencia de la traducción entre estas lenguas ha motivado, y también, las reflexiones que suscita la práctica traductora en el ejercicio de la profesión. De estas motivaciones son fruto los trabajos que integran los siguientes bloques y que estructuramos en los siguientes apartados: estudios históricos, estudios descriptivos sobre variedades de traducción y estudios aplicados. No podemos aspirar, sin embargo, a la exhaustividad, lo que sería un atrevimiento; más bien intentaremos dibujar los primeros trazos de un inventario que esperamos se lleve a cabo en el futuro con la minuciosidad que requiere. Incluiremos tanto los textos dedicados de manera específica a la combinación español-catalán, como los que hacen referencia a ella de manera tangencial o no específica.

\section{Los estudios históricos}

Los estudios históricos sobre la traducción entre español y catalán han interesado tanto a traductólogos como a historiadores o lingüistas. Dividimos el apartado en dos bloques, el primero lo integran los trabajos que se ocupan de la época medieval, y el segundo los dedicados a las últimas décadas del siglo xx y los primeros años del siglo XXI. Durante los siglos Xvi al XIX se interrumpe la práctica de esta traducción coincidiendo con la decadencia del catalán, por lo que también decae el interés investigador por este periodo.

\section{1. Época medieval}

Reseñamos en primer lugar el trabajo de Santoyo (1995) «El siglo XIV: traducciones y reflexiones sobre la traducción» de Santoyo (1995), en el que el autor revisa las traducciones realizadas a lo largo del siglo xiv en la Península Ibérica (desde lenguas como el latín, el árabe, el catalán, el castellano, el aragonés, etc.), un siglo poco estudiado en general desde el punto de vista de la traducción. El trabajo dedica una interesante parcela a las traducciones entre catalán y castellano, tanto a las traducciones directas, como a las indirectas o en cadena. El autor señala que «La lengua origen por excelencia durante todo el siglo xiv fue el latín, del cual en toda la Península se tradujo abundantísimamente, y mucho más aún al catalán que al castellano» (Santoyo 1995: 21); y muchas de estas traducciones del latín al catalán sirvieron, a su vez, de originales para la traducción de estos textos al castellano, como fue el caso del Llibre anomenat Valeri Màxim del original Dictorum factorumque memorabilium libri novem de Valerio Máximo. A partir de la versión en catalán se hicieron por lo menos tres traducciones al castellano durante el siglo $\mathrm{xv}$. $^{1}$

El trabajo de Santoyo se complementa en el mismo volumen con el de Ardemagni (1995) «The Role of Translation in Medieval Spanish and Catalan Literature», en el que la autora analiza el papel de la traducción en la España medieval y en la literatura catalana. En su opinión, los grandes autores de la literatura catalana de los siglos XIV 
y Xv mantuvieron su vitalidad en los siglos de decadencia a través de las traducciones al castellano:

Many of the authors from this period, such as Bernat Metge, Jordi de Sant Jordi, Ausiàs March, Jaume Roig and Joanot Martorell, were well-known to a Castilian-reading public. In the sixteenth century when Catalan literature fell into a period of decadence, the major works of the fourteenth and fifteenth centuries were kept alive through Castilian translations and the Catalan authors are therefore given the recognition that they deserve. (Ardemagni 1995: 75)

Dedicados a estos siglos están también los trabajos de Riera i Sans (1989) «Catàleg d'obres en català traduïdes en castellà durant els segles XIV i XV», Alvar (2010) Traducciones y traductores. Materiales para una historia de la traducción en Castilla durante la Edad Media, o Alvar y Lucía Megías (2009) Repertorio de traducciones al castellano del siglo $\mathrm{XV}$.

Alvar (2010) establece cinco grupos de traducciones en el fructífero siglo xv: traducciones directas, traducciones indirectas, traducciones inversas, autotraducciones y retraducciones. A pesar de que no se reseñan textos traducidos del catalán al español en los cinco grupos, el hallazgo de significativos ejemplos deja constancia de la importancia de la traducción entre catalán y español en aquel período. En el apartado dedicado a la traducción directa el autor explica que: «El catalán es la lengua original de las obras traducidas directamente por Miguel de Cuenca, Martín Martínez de Ampiés o fray Gonzalo de Ocaña» (2010: 244). Fray Miguel de Cuenca y fray Gonzalo de Ocaña, por ejemplo, tradujeron El llibre dels Àngels, de Francesc Eiximenis en 1434.

Respecto a las traducciones indirectas o en cadena sostiene que durante toda la Edad Media no fueron tan frecuentes como las traducciones directas pero, en el caso del catalán, sí han podido catalogarse en la combinación: latínà francésà catalánà castellano. Como la traducción del De civitate Dei de S. Agustín, por ejemplo, la cual pasó por el francés gracias a Raoul de Presles y del francés al catalán, y Gómez García del Castillo se ocupó de traducir el texto catalán al castellano. ${ }^{2}$

El volumen no da cuenta de las traducciones inversas, pero sí ofrece ejemplos de autotraducciones. Alvar cita el ejemplo de D. Enrique de Villena, que redactó en catalán Els dotze treballs d'Hèrcules, y luego lo vertió al castellano a instancias de Juan Fernández de Valera.

Finalmente, también muestra ejemplos de retraducciones en el itinerario: catalán-español-catalán. Uno de ellos es obra de Manuel Díez de Calatayud, que escribió El llibre del art de menescalia entre 1443 y 1450, y Martín Martínez de Ampiés realizó una versión al castellano, con reiteradas reimpresiones hasta el siglo XVI y, tras el éxito del esta traducción, se retradujo al catalán entre 1515 y 1530 (Alvar 2010: 250).

Los trabajos de Riera i Sans (1989) "Catàleg d'obres en català traduïdes en castellà durant els segles XIV i XV», y Alvar y Lucía Megías (2009) Repertorio de traducciones al castellano del siglo $\mathrm{XV}$, responden también al objetivo de compilar el inventario de las traducciones realizadas durante los siglos xIV y xv. Para Riera i Sans (1989), el listado de traducciones realizadas en los siglos xIV y xv (que supera las sesenta), es mucho más amplio de lo que nadie había sospechado (1989: 700). Entre los autores reseñados aparecen tanto nombres ampliamente conocidos como Aristóteles, Cicerón, Boecio, Petrarca, Arnau de Vilanova, Desclot, Eiximenis, Llull 
o Martorell, como autores o textos de menor repercusión: Bernat de Gordon, Pere Azemar, Manuel Dieç, el Art de Confessió o el Art de Bé Morir. El autor, con este trabajo, quiere poner de manifiesto la influencia que ha ejercido la cultura catalana sobre la castellana, al mismo tiempo que lamenta lo poco que se ha estudiado la traducción entre estas lenguas a pesar de la importancia que tuvo. Lo expresa así:

Les traduccions, entre moltes altres coses positives, són un índex ben eloqüent de la capacitat d'assimilació que té una llengua. Diu molt a favor del castellà que assimilés tantes coses bones que existien en aquella època posades en català. Per causes que no és ara el moment d'analitzar, però, tots els historiadors que dèiem, sense excepció, hi han passat de llarg. I continuen passant-hi, donant a entendre, implícitament, que no creuen que hagi existit mai una influència de la cultura catalana sobre la castellana, $\mathrm{i}$ fent veure que ignoren el fenomen de les traduccions entre aquestes dues llengües (Riera i Sans 1989: 699-700). ${ }^{3}$

Otro grupo de trabajos está compuesto por los dedicados a la obra de los grandes autores medievales, Ausiàs March, Martorell, Roís de Corella, Bernat Metge, etc. Ausiàs March, por ejemplo, ha sido ampliamente estudiado. Riquer, en Traducciones castellanas de Ausiàs March en la Edad de Oro (1946), recogía y estudiaba las traducciones al castellano de El Brocense, de Romaní, de Boscán, de Garcilaso, y la repercusión que tuvo la obra marquiana a través de estas traducciones. Una de ellas en concreto, la de Jorge de Montemayor, aparece publicada por Riquer en Ausiàs March, Poesías (1990b). Cabré en «Algunes imitacions i traduccions d'Ausiàs March al segle XVI» (2002), por su parte, valora el grado de fidelidad de las traducciones de Ausiàs March en función del momento literario y las modas imperantes y de acuerdo con la competencia lingüística y literaria de los traductores. Martines también se adentra en las traducciones al castellano de Ausiàs March en los textos: «La obra de Ausiàs March en el contexto de la lírica románica medieval» (2000), «Traducciones al servicio de la exégesis temprana sobre Ausiàs March y otros clásicos valencianos y el sentido de sus relaciones románicas» (2005), "Acaraments i contrastos literaris. Filologia i traducció per entendre Ausiàs March» (2006), o «Materials per a l'anàlisi multilingüe i contrastiva de locucions, col-locacions i fraseologia. Un escandall quant a Ausiàs March i Tirant lo Blanch» (2007), en los que realiza un análisis filológico de los originales y las traducciones y estudia la recepción de las obras de March a través de la traducción.

También la obra de Martorell, Tirant lo Blanc, conforma en sí misma un apartado específico. Riquer incluye un estudio sobre la traducción del Tirant lo Blanc en sus ediciones de 1974 y 1991, o en textos como «La difusió del Tirant lo Blanc» (1990). También se incluye un estudio sobre la traducción de la obra en las ediciones de Buendía (1954) y Vidal Jové (1969). En los trabajos de López Estrada (1993) «El Tirante castellano de 1511 y los libros de viajes», Mérida (1993) «¿Las desgracias de un editor? Diego de Gumiel, Tirant el Blanc y Tirante el Blanco», se estudia el contexto y la recepción editorial de la versión castellana del Tirante. Martines (1997), por su parte, en El Tirant poliglota, contrasta las traducciones española, italiana y francesa de los siglos XVI-XX.

De la obra traducida de Roís de Corella se ocupa también Martines (1999), en «Sin adobo se podrán bien servir'. Traducción y Filología: traducción al español de la lírica de Joan Roís de Corella», o investigadores del instituto ISIC-IVITRA (Instituto Superior de Investigación Cooperativa-Instituto Virtual Internacional de 
Traducción), como Fuster Ortuño «Curial e Güelfa multilingüe. Les traduccions del Curial e Güelfa al castellà $i$ a l'anglés. Concordances $i$ traduccions» (2007), o «La traducción de clásicos medievales: la novela caballeresca Curial e Güelfa» (2008).

Cabe destacar finalmente el trabajo de Tomás Martínez y Roxana Recio (2001) Essays on Medieval Translation in the Iberian Península, en el que se incluyen, entre otras, las contribuciones de Cifuentes (2001) «Las traducciones catalanas y castellanas de la Chirurgia magna de Lanfranco de Milán: un ejemplo de intercomunicación cultural y científica a finales de la Edad Media», o Hauf (2001) «Fray Hernando de Talavera, O.S.H., y las traducciones castellanas de la Vita Christi de Fr. Francesc Eiximenis, O.F.M.»; artículos en los que los autores insistn en el papel que jugó la traducción entre catalán y español en el avance científico de la época. En este último texto, en concreto, Cifuentes concluye que la traducción catalana de Lanfranco de Milán jugó un papel esencial en la formación de los cirujanos del siglo XIV de la Corona de Aragón. Tras el éxito de la traducción, el texto se importó a Castilla en diferentes traducciones al castellano, lo que permitió la incorporación de las nuevas técnicas quirúrgicas italianas por parte de los médicos de Castilla. De modo que «En el caso de la Chrirurgia magna de Lanfranco de Milán el papel de puente reconocido a la Corona de Aragón y al catalán tuvo un funcionamiento más complejo que el de una simple traducción» (Cifuentes 2001: 122).

En resumen, estos investigadores están llevando a cabo un trabajo destacable, que cubre una parcela específica de la combinación de lenguas objeto de estudio, las traducciones de la época medieval, un periodo de ingente publicación pero de difícil estudio. En palabras de Alvar (2010: 153), la historia de la traducción en España durante la Edad Media está por hacer, y no es trabajo fácil: la falta de materiales, la falta de relación entre unos textos y otros, el poco interés que han despertado las obras traducidas entre los estudiosos, son algunos de los escollos con los que se encuentra el investigador que se acerca a estos dominios. Conscientes de las dificultades son Lafarga y Pegenaute, quienes casi de manera simultánea a estas afirmaciones publican el Diccionario histórico de la traducción en España (2009); un trabajo riguroso en el que la época medieval ocupa un lugar destacado. La entrada «Traducción desde el catalán al castellano», de Chieregato y Gallén, es un excelente mapa de ruta de las traducciones del catalán al castellano desde sus inicios hasta el momento actual.

\subsection{Siglos $X X$ y XXI}

Tras el vacío que supusieron los tres siglos de decadencia de las letras catalanas, y con ellas de la traducción -con las excepciones de las traducciones de temática religiosa o las traducciones cotidianas (Santoyo 1999)-, hemos de esperar hasta el momento presente para que el estudio de la traducción entre catalán y español despierte de nuevo interés. El segundo periodo estudiado desde planteamientos históricos es por lo tanto el siglo xx y primera década del siglo xxI.

Destacamos en primer lugar el trabajo de Romaguera (1988) «Traduccions entre llengües de l'Estat español», al tratarse del primer texto que se ocupa de manera específica de la traducción entre el español y el catalán durante el siglo xx. En este trabajo, a partir de datos sobre la producción editorial, el autor analiza los diferentes momentos de producción e infiere las diferentes tendencias observadas en la política editorial tanto en la dirección del catalán al castellano como del castellano al catalán; 
sin olvidar tampoco las ediciones bilingües o las traducciones del euskera o el gallego al catalán, y del catalán a estas lenguas, ni la catalogación de las obras en función del género literario al que pertenecían.

Ofrecen también un recorrido histórico centrado en el siglo xx los trabajos de Bacardí «La traducció del castellà al català al segle $\mathrm{xx}$, esbós d'una història accidentada» (2001a), y «Translation from Spanish into Catalan during the 20th century: sketch of a chequered history» (2004). En el primero de ellos, la autora inicia el recorrido en la Edad Media. Esta revisión histórica le permite ofrecer las claves que explican la situación actual, es decir, por qué se traduce al catalán en la actualidad para unos lectores que dominan ya perfectamente el castellano y pueden leer la obra en la lengua original. El trabajo representa un primer esfuerzo loable de comenzar la tarea de recopilación de lo que puede constituir una historia de la traducción entre estas lenguas, y se ve enriquecido con reflexiones de tipo sociológico. En el artículo de 2004 «Translation from Spanish into Catalan during the 20th century: sketch of a chequered history», la autora realiza una cartografía de las obras y autores traducidos desde principio del siglo $\mathrm{xx}$.

El trabajo de Sellent (1998) «La traducció literària en català al segle xx: alguns títols representatius», a pesar de ser un trabajo fundamental para una visión exhaustiva y crítica de la traducción (y de los traductores) al catalán del siglo xx, de sus dependencias y libertades, no es, sin embargo, específico sobre la traducción entre castellano y catalán. Solo de manera tangencial incluye referencias y estas tienen que ver más con la producción editorial que con la traducción. El autor sostiene que las traducciones al catalán de los últimos años y su merma de calidad responden en muchas ocasiones al afán de editarlas con rapidez antes de que aparezca la traducción al castellano.

Finalmente, también centrados en la evolución reciente de la traducción en el ámbito editorial, contamos con los textos de Lafarga «Empuje cultural y freno económico: las traducciones literarias al castellano y al catalán en competencia» (2004), $\mathrm{y}$ «Sobre la traducción en Cataluña en época reciente: cultura, comercio, competencia de lenguas» (2006).

Siguen también un recorrido histórico los textos de Bacardí y Estany (1999) «La mania cervàntica. Les traduccions del Quixot al català (1836-50?-1906)», Bacardí (2001b) «Traduir el Quixot al català: més enllà de la intel-ligibilitat», a los que remitiremos más adelante en el apartado sobre estudios de traducción literaria.

\section{Estudios sobre variedades de traducción}

En este apartado solo podemos reseñar trabajos dedicados a algunas variedades concretas de traducción: la traducción jurídico-administrativa, la traducción literaria, la traducción bíblica y, más tímidamente, la traducción publicitaria. Otras, sin embargo, como la traducción audiovisual o la traducción científica no han recibido atención por parte de los investigadores, lo cual no es de extrañar si tenemos en cuenta que la práctica profesional de la traducción entre español y catalán es una práctica desigual en estos ámbitos profesionales. Remitimos a un trabajo precedente, García de Toro (2004) «Translation between Spanish and Catalan today», en el que se observan las tendencias de traducción entre castellano y catalán en los diferentes ámbitos de especialidad en las últimas décadas. 


\subsection{Textos jurídico-administrativos}

La traducción jurídico-administrativa es la variedad que mayor reflexión ha suscitado. Después de ser reconocida la cooficialidad de las lenguas en el año 1978, la necesidad de garantizar y preservar su uso en los diferentes ámbitos públicos justifica la traducción de la documentación administrativa y jurídica. Y, sin entrar a valorar cómo y hasta dónde se han cumplido estos objetivos, lo que sí constatamos es que se publican una serie de trabajos preocupados fundamentalmente por los problemas lingüísticos y textuales de la traducción de estos documentos, especialmente del castellano al catalán. Buena parte de ellos son obra de Duarte:

- El català llengua de l’administració (1980).

- Curs de llenguatge administratiu (1981).

- «Lenguas en contacto: el estándar y el lenguaje administrativo» (1982).

- «Els problemes del llenguatge administratiu català» (1984a) .

- «Els nivells d'influència castellana en el llenguatge administratiu català» (1984b).

- «Criteris per a la traducció administrativa entre el català i el castellà» (1984c).

- «Llengua i administració. Estudis sobre la llengua el dret i l’administració» (1986).

- «Introducció a la traducció jurídica i administrativa» (1993).

- «El llenguatge administratiu i jurídic: la necessitat d’un nou pas endavant» (2002).

- Manual de llenguatge administratiu (1991) en colaboración con Alsina y Sibina.

Son trabajos que responden a una preocupación fundamental: contribuir a la normalización del lenguaje administrativo y jurídico en catalán y conseguir que el catalán se convierta en una lengua apta para el uso social.

Así, en uno de los textos clave de este apartado, el de Duarte (1993) «Introducció a la traducció jurídica i administrativa», el autor combina la descripción de las características definitorias de la traducción administrativa con la tipificación de los problemas de traducción más frecuentes y sus posibles soluciones (topónimos, designaciones de cargos, organismos, instituciones, siglas, títulos de leyes o disposiciones, fraseologismos, etc.). Incluye también comentarios de tipo contrastivo encaminados a la eliminación de errores. Veamos alguno de ellos:

Pel que fa a les qüestions gramaticals, i exemplificant-ho també en el cas del francés i del castellà, cal evitar en traduccions al català elisions de la conjunció que per influència d'una construcció habitual -i gens recomanable en castellà- («SOL.LICITA li sigui concedit permís» en lloc de «SOL·LICITA que li sigui concedit permís»), construccions amb gerundi especificatiu (tan freqüent en francés i també present en castellà, per exemple: «instancia solicitando permiso»), un ús excessiu de la passiva amb ésser [...], construccions de previsió-obligació amb la preposició $a$ (generals en francés i freqüents, per influència del francés, en castellà: «mesures à appliquer» per «mesures que cal aplicar») [...] Cal, doncs, garantir un ple respecte a la gramàtica de la llengua d'arribada a l'hora de fer una traducció i evitar d'introduir solucions gramaticals que reprodueixen models de la llengua de partença quan no siguin vàlids en la llengua d'arribada. (Duarte 1993: 96)

Estas obras nacen con una voluntad de practicidad incuestionable y en un momento, los años 80 y principios de los 90, de intenso trabajo en el campo de la normalización de la documentación administrativa en catalán. Sirvieron para llenar un vacío existente, ya que toda la documentación estaba en castellano, y se puede decir que cumplieron con creces la función asignada. 
Otros trabajos en esta misma línea son las publicaciones de instituciones como la Generalitat de Cataluña o la Generalitat Valenciana (y de sus departamentos de política lingüística), o de los gabinetes y servicios de lenguas de las diferentes universidades, aunque desgraciadamente en algunos casos son de difusión interna (como el texto de Generalitat de Catalunya (1999) «Criteris de traducció de textos normatius del castellà al català» (1999), que se difundió con una tirada de 5.000 ejemplares).

- Generalitat Valenciana (1984): Manual de llenguatge administratiu valencià.

- Generalitat Valenciana (1990): L'ús del valencià en l'administració autonómica.

- Universitat de Barcelona (1991): DAU Documents administratius universitaris.

- Universitat Jaume I (2003): Manual de documents i llenguatge administratius.

- Universitat Pompeu Fabra (2004): Documents administratius de la Universitat Pompeu Fabra.

- Universitat de València (2007): «Criteris lingüístics per als usos institucionals de les universitats valencianes».

En general son trabajos que recogen el quehacer diario de los traductores de los servicios lingüísticos mencionados y constituyen una referencia obligada para los traductores interesados en esta variedad de traducción. Prestan especial atención a los contactos lingüísticos con el castellano e incluyen los aspectos gramaticales más problemáticos: orden de los elementos de la frase, anáforas y nominalizaciones, construcciones verbales perifrásticas, etc., además de cuestiones terminológicas y fraseológicas. En algunos casos incluyen listados terminológicos en las dos lenguas y ofrecen pautas de construcción textual y genérica a partir de la observación y el trabajo con este tipo de textos. La mayor parte de ellos, sin embargo, no están dedicados de manera específica a la traducción, sino a la redacción en catalán.

\subsection{Textos literarios}

Mencionaremos en primer lugar los trabajos que se incluyen en el volumen de Bacardí, Fontcuberta y Parcerisas (1998) Cent anys de traducció al català (1891-1990) Antologia:

- Bulbena y Tusell (1891): «Pròlech». En: Miquel de Cervantes Saavedra. L'enginyós cavaller don Quixot de la Manxa.

- Rullán (1905): «Cuatre paraules d'es autor a n’es lectors». En: Miquel de Cervantes Saavedra. L'enginyós hidalgo don Quixote de la Mancha.

- Llompart (1966) «Pròleg del traductor». En:Camilo José Cela. Viatge al Pirineu de Lleida.

Se trata de los prólogos a las traducciones de estas obras del español al catalán donde se exponen algunos de los criterios de traducción seguidos: la supresión de repeticiones en el primero, la eliminación de los arcaísmos en el segundo, o la introducción de mallorquinismos en la traducción del libro de Cela.

En este apartado cabe mencionar también otro grupo de trabajos, los dedicados a la reflexión sobre la autotraducción, entre los que contamos con el de Carme Riera (1997) «L'autotraducció com a exercici de recreació», o Antoni Marí (1997) «L'experiència de l'autotraducció». Estos dos escritores reflexionan sobre sus incursiones en la traducción del catalán al español como traductores de sus propias obras, escritas originariamente en catalán. Y de sus observaciones se desprende que el ejercicio de 
la autotraducción, como práctica asociada al bilingüismo, se convierte en muchas ocasiones en un ejercicio de creación, de reescritura. Carme Riera, a propósito de su traducción de Dins el darrer blau /En el último azul, comenta que en muchas ocasiones, aunque estuviera escribiendo y contando la misma historia, lo hacía de manera diferente: hay párrafos que han desaparecido, capítulos más resumidos, situaciones que han cambiado un poco, o incluso se han solucionados errores que después han permitido mejorar el texto original en las futuras ediciones.

Otro grupo de trabajos lo conforman los dedicados a la traducción de la obra clave de la literatura española, Don Quijote de la Mancha. Entre ellos hay que destacar los ya mencionados de Bulbena y Tusell (1891) o Rullán (1905) o los de Bacardí y Estany (1999) «La mania cervàntica. Les traduccions del Quixot al català (1836-50?1906)», Bacardí (2001b) «Traduir el Quixot al català: més enllà de la intel-ligibilitat». Estos dos últimos imbrican la vertiente histórica y la sociológica en el análisis de la recepción de estos textos.

Otro trabajo, centrado ahora en la literatura juvenil, es el de la autora de estas páginas, García de Toro (2005) «Tendencias de traducción entre lenguas en contacto en la literatura juvenil». Y de reciente aparición es el trabajo de Bacardí y Godayol (2011) Diccionari de la traducció catalana, un catálogo de traductores, traducciones y material paratextual y crítico sobre la traducción catalana, un ingente trabajo en el que han colaborado más de ochenta investigadores. La obra compila por primera vez la lista de traductores más relevantes de todos los tiempos, hasta los nacidos en 1950, que han traducido desde cualquier lengua al catalán, así como las traducciones anónimas y colectivas más sobresalientes. Una obra de referencia, pionera y original, de la que muy pocas lenguas disponen y que permite extraer datos clave sobre los traductores y la traducción del español y catalán y a la inversa.

Finalmente, cabe reseñar los trabajos que incluyen referencias a la traducción catalán/castellano de manera tangencial. En muchos casos se trata de comentarios sobre la traducción al catalán pero desde diferentes lenguas, y no específicamente desde el castellano; entre ellos cabe señalar el de Vallverdú (1998) «Els problemes de la traducció», en el que incluye los datos estadísticos sobre las traducciones al catalán desde todas las lenguas, o Mallafrè (1991) Llengua de tribu i llengua de polis: bases d'una traducció literaria, en el que el autor clasifica los problemas del traductor literario, e incluye ejemplos en las dos lenguas, fundamentalmente de problemas lingüísticos y culturales.

\subsection{Textos bíblicos}

De la traducción bíblica se han ocupado Morreale (1958) «Apuntes bibliográficos para la iniciación al estudio de las traducciones bíblicas medievales en catalán», o Puig i Tàrrech (1987) «Les traduccions bíbliques catalanes en el segle XIX».

Morreale ofrece una revisión bibliográfica de las traducciones bíblicas medievales al catalán y, aunque no es el objetivo, compara la traducción al catalán y al castellano al establecer las diferencias entre los primitivos textos bíblicos. El estudio arroja interesantes conclusiones como las siguientes: 1) La Vulgata penetró más pronto y más profundamente en la cultura catalana que en la castellana; 2) las versiones españolas de la Biblia se hicieron directamente del texto hebreo, mientras que la Biblia catalana tiene su origen en la Vulgata; 3) faltan versiones hebreo-catalanas; 4) en la 
historia de la Biblia, Cataluña sirve de puente entre el ámbito castellano y el francoprovenzal en la transmisión de los textos latinos. ${ }^{5}$

El artículo de Puig i Tàrrech analiza las traducciones bíblicas catalanas del siglo $\mathrm{XIX}$, e incluye comentarios sobre sus diferencias con la traducción al castellano, como en el siguiente:

Per als eclesiàstics del primer terç del s. XIX, el castellà és la llengua culta i pròpiament il.lustrada. Les edicions en català tenen la funció exclusiva de nodrir la fe del poble que no entén el castellà, és a dir, la gent sense instrucció ni cultura; o bé ajudar els clergues, que tenen coneixements bíblics migrats, en la seva missió de predicació. (Puig i Tàrrech 1987: 99 $)^{6}$

Pero ninguno de estos trabajos trata sobre aspectos específicos de la traducción entre catalán y español sino que introducen comentarios dispersos, en su mayoría de tipo sociológico (contrastan las diferencias de acogida de los textos bíblicos en las dos lenguas), o histórico-filológico (buscan los orígenes diferentes de la Biblia catalana y castellana).

\subsection{Textos publicitarios}

En este apartado encontramos el trabajo de Sabaté (1999) La publicitat en català. Está dedicado a la publicidad en catalán pero se ocupa de la traducción en uno de los capítulos del libro. Para Sabaté, la traducción de la publicidad al catalán se realiza desde el castellano por lo que es subsidiaria de esta. La práctica cotidiana determina que las campañas en catalán sean mayoritariamente traducciones del castellano, sea ésta la lengua de origen o la lengua intermediaria y si lo que se anuncia es tanto un producto nacional como extranjero. La traducción de la publicidad al catalán se ve condicionada y fuertemente determinada por las soluciones que han aparecido y han penetrado en el público hispanohablante. A partir de esta constatación, el autor establece una tipificación de los principales problemas que se presentan al traductor -juegos de palabras, homofonías, elementos intertextuales, problemas derivados de las limitaciones del estándar catalán, etc.-, y de cómo han sido resueltos: transformaciones conceptuales, aparición de elementos en castellano, barbarismos, arcaísmos, cambios de registro, etc.

\section{Los estudios aplicados}

En este apartado se incluyen los trabajos dedicados a la crítica y comparación de traducciones así como a la didáctica de la traducción.

\subsection{Crítica y comparación de traducciones}

Las contribuciones que hemos podido reseñar dedicadas específicamente a la traducción entre catalán y español son las de Bordoy «Ildefonso Rullán, traductor del Quijote al mallorquín» (1952), y La traducción mallorquina del 'Quijote' (1956), ambas centradas en la traducción del Quijote a la variedad mallorquina del catalán.

No dedicados específicamente a la traducción entre catalán y castellano están los trabajos de Parra i Albà (1995) «Adaptaciones teatrales de Los tres mosqueteros de Alejandro Dumas», en el que se comparan las adaptaciones catalana de 1977 y la 
castellana de 1939 de esta obra de Dumas; el de Sánchez Rodrigo y Nogueras (1996) «Els poetes com a traductors: Kavafis en català, Kavafis en castellà», en el que se analizan las traducciones de Kavafis a estas lenguas, y se concluye con una valoración más positiva de la traducción al catalán que al castellano; o el de Kenfel y Lorenzo (2003) Estudios críticos de traducción de literatura infantil y juvenil. Análisis de las traducciones de obras inglesas y alemanas a las cuatro lenguas oficiales de España, en el que se comparan las traducciones de un mismo original a las cuatro lenguas del estado: gallego, catalán, euskera y castellano.

\subsection{La didáctica de la traducción}

La didáctica de la traducción entre estas lenguas es todavía incipiente. ${ }^{7}$ Los primeros trabajos aparecen tras la implantación de la licenciatura de Traducción e Interpretación en las universidades del Estado español en las que se imparten asignaturas de traducción catalán-español: Universitat d'Alacant, Universitat Autònoma de Barcelona, Universitat Jaume I, Universitat Oberta de Catalunya, Universitat Pompeu Fabra, Universitat de Valencia, Universitat de Vic.

La primera tentativa es obra de Fontcuberta (1982) «La traducción y el contacto de lenguas. Algunas consideraciones». El autor, movido por intereses didácticos, reflexiona sobre la situación del traductor y del aprendiz de traductor en un contexto de lenguas en contacto como el que se vive en Cataluña. Diferencia entre las habilidades lingüísticas de los hablantes bilingües y las de los traductores, y reflexiona sobre el nivel de conocimiento de la lengua de partida y de la lengua de llegada que debe tener un traductor. Considera que, en el contexto de lenguas en contacto, un paso previo para el estudiante de traducción es separar correctamente las lenguas, para lo cual aconseja afianzar la lengua materna. Advierte finalmente de los problemas que provoca la diglosia, como son los calcos y los préstamos, los cuales conducen a barbarismos.

Por su parte, el trabajo de García de Toro y Hurtado (1999) «La traducción entre lenguas maternas», está dedicado al establecimiento de los objetivos de aprendizaje y la metodología específica para la enseñanza de la traducción entre español y catalán. Las particularidades de la enseñanza de este tipo de traducción, derivadas fundamentalmente de la cohabitación de las lenguas y de su proximidad formal, aconsejan un diseño de los objetivos que contemple las peculiaridades del mercado laboral: la bidireccionalidad que se solicita a los profesionales o la amplitud de los campos de especialización.

Paralelamente a esta publicación, aparecen ya los primeros manuales didácticos y textos de materiales docentes, como los de García de Toro (1998) Traducción catalán-español (II). Cuaderno de trabajo, o Agost y Monzó (2001) Teoría y práctica de la traducción general espanyol-català. El primero se compone de materiales estructurados sobre la clasificación de problemas de traducción lingüístico-textuales, a partir de los planteamientos de Baker (1992) y Hatim y Mason (1999); y en el volumen de Agost y Monzó los materiales se estructuran en función de las subcompetencias que integran la competencia traductora a partir de los postulados del grupo PACTE (2000, 2001).

De reciente aparición son los trabajos de Doménech (2012) «Traduir del castellà al català. Més enllà de la intercomprensió», y el capítulo de la misma autora en el 
volumen colectivo de Bacardí, Doménech, Gelpí, et al. (2012) Teoria y pràctica de la traducció. En ellos la autora se centra en la traducción administrativa del español al catalán, y en los puntos conflictivos de los planos gramatical y estilístico: los gerundios, la voz pasiva, el futuro de obligación, las mayúsculas y minúsculas, las abreviaturas, etc.

\section{Recapitulación y prospectiva}

Los trabajos presentados en este artículo nos permiten concluir que todos ellos van conformando ya un nuevo panorama investigador. Quedan ya representadas las ramas descriptiva y aplicada de la disciplina, aunque tímidamente en algunos casos y con las carencias propias de un panorama incipiente. Los ámbitos mejor representados hasta el momento son los siguientes:

1. Los estudios históricos: tanto los centrados en la época medieval, como en los siglos XX y XXI.

2. Los estudios dedicados a las variedades de traducción como la traducción jurídica y administrativa, la traducción literaria, la traducción de textos bíblicos o la traducción de textos publicitarios.

3. Los estudios aplicados: preocupados esencialmente por la didáctica de la traducción.

Después de estas primeras fases de trabajos descriptivos y aplicados y de la consolidación de los estudios históricos, de más larga tradición, es necesario que el panorama investigador se amplíe. Se observa una gran desatención en ámbitos como la traducción audiovisual (la subtitulación, el doblaje, la traducción para la accesibilidad), o la traducción de productos informáticos. Y el proceso se debería completar con estudios empírico-experimentales, centrados en el proceso cognitivo de la traducción entre estas lenguas y en el grado de bilingüismo de los traductores. Pensamos que este tipo de estudios puede abrir una vía de investigación atractiva, puesto que la medición y comparación de los niveles de bilingüismo en el proceso de traducción es quizás uno de los campos de análisis en los que la traducción entre catalán y español puede aportar datos de especial relevancia.

Para concluir, solo comentar que este trabajo queda necesariamente abierto. Esperamos que futuros investigadores tomen el testigo de lo que aquí es solo un esbozo de la investigación entre catalán y español y completen así el mapa de ruta y contribuyan a su tan necesaria visibilidad.

\section{NOTAS}

1. Avenoza (2001: 47) corrobora este dato y concreta que la traducción al castellano de Juan Alfonso de Zamora procede de la traducción catalana de Antoni Canals.

2. La función de estas traducciones en cadena ha sido analizada por Wittlin (1995: 4). El autor sostiene que permiten documentar el estimulante papel de puente que la lengua y la cultura catalanas ejercieron sobre la castellana en un momento histórico en que, por circunstancias de todos conocidas, la cultura catalana se abrió completamente a los aires europeos.

3. Las traducciones, entre otras muchas cosas positivas, son un indicio muy elocuente de la capacidad de asimilación que tiene una lengua. Dice mucho a favor del castellano que asimilara tantas cosas buenas que existían en aquella época puestas en catalán. Sin embargo, por causas que no es ahora el momento de analizar, todos los historiadores que comentábamos, sin excepción, han pasado de largo. Y continúan pasando, dando a entender, implícitamente, que no creen que haya existido nunca una influencia de la cultura catalana sobre la castellana, y haciendo ver que ignoran el fenómeno de las traducciones entre estas dos lenguas. (Riera i Sans 1989: 699-700, traducción de la autora) 
4. Con respecto a las cuestiones gramaticales, y ejemplificándolo también en el caso del francés y del castellano, hay que evitar en traducciones al catalán elisiones de la conjunción que por influencia de una construcción habitual -y nada recomendable en castellano- («SOL.LICITA li sigui concedit permís» en lugar de «SOL·LICITA que li sigui concedit permís»), construcciones con gerundio especificativo ( $\tan$ frecuente en francés y también presente en castellano, por ejemplo: «instancia solicitando permiso»), un uso excesivo de la pasiva con ser [...], construcciones de previsiónobligación con la preposición $a$ (generales en francés y frecuentes, por influencia del francés, en castellano: «mesures à appliquer» por «mesures que cal aplicar») [...] Hay que garantizar un pleno respeto a la gramática de la lengua cuando se hace una traducción y evitar introducir soluciones gramaticales que reproducen modelos de la lengua de partida cuando estos no sean válidos en la lengua de llegada. (Duarte 1993: 96, traducción de la autora).

5. La autora, al tratar aspectos tan polémicos como es el origen de la Biblia catalana, encuentra su réplica. Perarnau (1978), por ejemplo, desmiente en parte estas afirmaciones al sostener que también existen textos catalanes de origen hebreo e incluso híbridos. En su opinión, en la Edad Media las traducciones al catalán se realizan tanto desde el hebreo como desde el latín (la Vulgata), y en la mayor parte de los casos a partir de textos híbridos.

6. Para los eclesiásticos del primer tercio del s. XIX, el castellano es la lengua culta y propiamente ilustrada. Las ediciones en catalán tienen la función exclusiva de nutrir la fe del pueblo que no entiende el castellano, es decir, la gente sin instrucción ni cultura; o bien ayudar a los clérigos, que tienen limitados conocimientos bíblicos, en su misión de predicación. (Puig i Tàrrech 1987: 99, traducción de la autora).

7. Los trabajos dedicados a la didáctica de lenguas que han incluido cuestiones de traducción entre estas lenguas han sido comentados en el artículo dedicado a los estudios lingüísticos (aparecido en esta revista con el título: «La investigación sobre la traducción entre catalán y español I: estudios lingüísticos»), por su adscripción a este ámbito y no propiamente al de la traducción. Es el caso del trabajo de Tió (1982), y en ciertos aspectos de los de Rodríguez-Vida (1997) y Jordana (1933). Como veíamos, eran trabajos concebidos fundamentalmente como gramáticas contrastivas y motivados por intereses didácticos. En los tres casos, por otro lado, la función de la traducción respondía a la concepción de ésta como una herramienta más dentro de la didáctica tradicional de lenguas.

\section{REFERERENCIAS}

Agost, Rosa y MONZÓ, Esther (2001): Teoria i pràctica de la traducció general espanyol-català. Castelló de la Plana: Universitat Jaume I.

Altés, Núria y Pejó, Xavier (2001): Manual de redacció administrativa. Vic: Eumo.

Alvar, Carlos (2010): Traducciones y traductores. Materiales para una historia de la traducción en Castilla durante la Edad Media. Alcalá de Henares: Centro de Estudios Cervantinos.

Alvar, Carlos y Lucía Megías, José Manuel (2009): Repertorio de traducciones al castellano del siglo XV. Madrid: Ollero y Ramos.

Ardemagni, Enrica (1995): The Role of Translation in Medieval Spanish and Catalan Literature. In: Roxana RECIO, ed. La traducción en Espana, ss. XIV-XVI. León: Universidad de León, 71-78.

Avenoza, Gemma (2001): Antoni Canals, Simon de Hesdin, Nicolás de Gonesse, Juan Alfonso de Zamora y Hugo Urriés: Lecturas e interpretaciones de un clásico (Valerio Máximo) y de sus comentaristas (Dionisio de Burgo Santo Sepulcro y Fray Lucas). In: Tomás Martínez y Roxana Recio, eds. Essays on Medieval Translation in the Iberian Península. CastellóOmaha: Univesritat Jaume I-Creighton University, 45-73.

BACARDí, Montserrat (2001a): La traducció del castellà al català al segle xx, esbós d'una història accidentada. (Conferencia). V Congrés Internacional de Traducció. Barcelona: Universitat Autònoma de Barcelona.

BACARDí, Montserrat (2001b): Traduir el Quixot al català: més enllà de la intel-ligibilitat. Revista de Catalunya. 165:70-78.

BACARdí, Montserrat (2004): Translation from Spanish into Catalan during the $20^{\text {th }}$ century: Sketch of a chequered history. In: Albert Branchadell y Margaret West, eds. Less Translated Languages. Amsterdam: John Benjamins, 257-268. 
Bacardí, Montserrat, Doménech, Ona, Gelpí, Cristina, et al. (2012): Teoría i pràctica de la traducció. Barcelona: Editorial UOC.

BACARDí, Montserrat y Estany, Inma (1999): La mania cervàntica. Les traduccions del Quixot al català (1836/50?-1906). Quaderns. Revista de Traducció. 3:49-59.

Bacardí, Montserrat, Fontcuberta, Joan y Parcerisas, Francesc, eds. (1998): Cent anys de traducció al català (1981-1990). Antologia. Vic: Eumo.

Bacardí, Montserrat y Godayol, Pilar, coord. (2009): Una impossibilitat possible. Trenta anys de traducció als Països Catalans (1975-2005). Vilanova i la Geltrú: Argumenta.

BACARDí, Montserrat y GodAYol, Pilar, dir. (2011): Diccionari de la traducció catalana. Barcelona: Eumo.

Badia i Margarit, Antoni Maria (1979): Formulari administratiu aplicat especialment a la universitat. $4^{\mathrm{a}}$ ed. Barcelona: Curial.

BAKer, Mona (1992): In Other Words. A Coursebook on Translation. Londres/ Nueva York: Routledge.

Bassols, Margarida, Rico, Albert y Torrent, Anna M. eds. (1997): La llengua de TV3. Barcelona: Empúries.

Bordoy, Miguel (1952): Ildefonso Rullán, traductor del Quijote al mallorquín. Anales Cervantinos. 2:427-430.

Bordoy, Miguel (1956): La traducción mallorquina del Quijote. Panorama Balear. 55:9-10

Buendía, Felicidad, ed. (1954): Libros de caballería españoles: El Caballero Zifar, Amadís de Gaula, Tirante el Blanco. Madrid: Aguilar.

Bulbena i Tusell, Antoni (1891): Pròlech. Miguel de Cervantes. L'enginyós cavaller don Quixot de la Manxa (1891). In: Montserrat BaCardí, Joan Fontcuberta y Francesc Parcerisas. Cent anys de traducció al català (1981-1990). Antologia. Vic: Eumo, 17-20.

CABRÉ, Lluís (2002): Algunes imitacions i traduccions d'Ausiàs March al segle XVI. Quaderns. Revista de traducció. 7:59-82.

CASAls, Daniel (2003): El català en antena. Vint anys construint el model lingüístic de Catalunya Ràdio. Benicarló: Onada Edicions.

Casanova, Emili (1980): Castellanismos y su cambio semántico al penetrar en catalán. Boletín de la Asociación Europea de Profesores de Español. 13:15-25.

Catford, John Cunnison (1965): A Linguistic Theory of Translation: an Essay on Applied Linguistics. Londres: Oxford University Press.

Chaume, Frederic y García de Toro, Cristina (2010): Teories actuals de la traductologia. Alzira: Bromera.

Chieregato, Chiara y Gallén, Enric (2009): Traducción desde el catalán al castellano. In: Francisco Lafarga y Luis Pegenaute, 200-205.

Cifuentes, Lluís (2001): Las traducciones catalanas y castellanas de la Chirurgia magna de Lanfranco de Milán: un ejemplo de intercomunicación cultural y científica a finales de la Edad Media. In: Tomás Martínez y Roxana Recio. Essays on Medieval Translation in the Iberian Península. Castelló-Omaha: Univ. Jaume I/Creighton Univ, 95-127.

Colón, Germà (1976): El léxico catalán en la Romania. Madrid: Gredos.

Delisle, Jean (1980): L’analyse du discourse comme méthode de traduction. Ottawa: Éditions de l'Université d'Ottawa.

DONÉNECH, Ona (2012): Traducir del castellà al català: més enllà de la intercomprensió. Barcelona: Editorial UOC.

Duarte, Carles (1980): El català llengua de l'administració. Barcelona: Indesinenter.

Duarte, Carles (1981): Curs de llenguatge administratiu. $3^{\mathrm{a}}$ ed. Barcelona: Teide.

Duarte, Carles (1982): Lenguas en contacto: el estándar y el lenguaje administrativo. HerriArduralaritzazko Euskal Aldizkaria. 4:117-120.

DuARte, Carles (1984a): Els problemes del llenguatge administratiu català. Serra d'Or. 294:11-12.

DuARTE, Carles (1984b): Els nivells d'influència castellana en el llenguatge administratiu català. Llengua i Administració. 11:8.

DuARTE, Carles (1984c): Criteris per a la traducció administrativa entre el català i el castellà. Llengua i Administració. 12:5. 
DuARTE, Carles (1986): Llengua i administració. Estudis sobre la llengua, el dret i l'administració. II Congrés Internacional de la Llengua Catalana. Barcelona: Edicions de la Magrana.

Duarte, Carles (1993): Introducción a la traducción jurídica y administrativa. In: Carles Duarte, Llengua i Administració. Barcelona: Columna, 88-111.

DuARTE, Carles (2002): El llenguatge administratiu i jurídic: la necessitat d'un nou pas endavant. Revista de Llengua i Dret. 38:13-17.

Duarte, Carles, Alsina, Àlex y Sibina, Segimon (1991): Manual de llenguatge administratiu. $6^{a}$ ed. Barcelona: Generalitat de Catalunya, Escola d'Administració Pública.

El Periódico de Catalunya (2002): Llibre d'estil. Barcelona: Ediciones Primera Plana.

Fontcuberta, Joan (1982): La traducció i el contacte de llengües. Algunes consideracions. Quaderns de Traducció i Interpretació. 1:29-37.

Fuster Ortuño, M. Àngels (2007): Curial e Güelfa multilingüe. Les traduccions del Curial e Güelfa al castellà $i$ a l'anglés. Concordances i traduccions. In: Instituto Alicantino de Cultura Juan Gil-Albert, Premios de Ayuda a la Investigación 2004. Alicante: Instituto de Cultura Juan Gil Albert - Excma. Diputación de Alicante, 38-88.

Fuster Ortuño, M. Àngels (2008): La traducción de clásicos medievales: la novela caballeresca Curial e Güelfa. In: Assumpta Camps y Lew Zyвatow, eds. La Traducción literaria en la época contemporánea. Actas de la Conferencia Internacional «Traducción e Intercambio Cultural en la Época de la Globalización», mayo de 2006, Universidad de Barcelona. Frankurt am Main: Peter Lang, 153-166.

García De Toro, Cristina (1998): Traducció català-espanyol II. Quadern de treball. Castelló de la Plana: Publicacions de la Universitat Jaume I.

García de Toro, Cristina (2004): Translation between Spanish and Catalan today. In: Albert Branchadell y Margaret West, eds. Less Translated Languages. Amsterdam: John Benjamins, 269-286.

García DE Toro, Cristina (2005): Tendencias de traducción entre lenguas en contacto en la literatura juvenil. AILIJ, Anuario de Investigación en Literatura infantil y juvenil. 3:97-134.

García de Toro, Cristina (2009): La traducción entre lenguas en contacto. Catalán y español. Berna: Peter Lang.

GARCíA DE TORO, Cristina (2015): La investigación sobre la traducción entre catalán y español: estudios lingüísticos. Meta 60(1):71-89.

García de Toro, Cristina y Hurtado, Amparo (1999): La enseñanza de la traducción entre lenguas maternas. In: Amparo HurTado, dir. Enseñar a traducir, Metodología en la formación de traductores e intérpretes. Madrid: Edelsa, 121-137.

Generalitat de Catalunya, Departament de cultura (1999): Criteris de traducció de textos normatius del castellà al català. Barcelona: Generalitat de Catalunya.

Generalitat Valenciana (1984): Manual de llenguatge administratiu valencià. Valencia: Conselleria de Cultura, Educació i Ciència.

Generalitat Valenciana (1990): L'ús del valencià en l'administració autonómica. Valencia: Conselleria de Cultura, Educació i Ciència.

Grellet, Françoise (1991): Apprendre à traduire. Nancy: Presses Universitaires de Nancy.

Guillemin-Flescher, Jacqueline (1981): Syntaxe comparée du français et de l'anglais. Problèmes de traduction. París: Ophris.

Hatim, Basil y Mason, Ian (1990): Discourse and the Translator. Londres: Longman.

Hauf, Albert (1995) Pròleg. In: Curt Wittlin. De la traducció literal a la creació literaria. Valencia/ Barcelona: Institut Interuniversitari de Filologia Valenciana/ Publicacions de l'Abadia de Montserrat.

Hauf, Albert (2001): Fray Hernando de Talavera, O.S.H., y las traducciones castellanas de la Vita Christi de Fr. Francesc Eiximenis, O.F.M. In: Tomás Martínez Romero y Roxana Recio. Essays on Medieval Translation in the Iberian Península. Castelló-Omaha: Univ. Jaume I/Creighton Univ, 203-250.

Hurtado, Amparo (2001): Traducción y Traductología. Introducción a la Traductología. Madrid: Cátedra. 
Intravaia, Pietro y Scavée, Pierre (1979): Traité de stylistique comparée du français et de l'italien. Paris: Didier.

JANÉ, Albert (1967): Gramàtica essencial de la llengua catalana. Barcelona: Bruguera.

Jordana, Carles (1933): El català i el castellà comparats. (Edición de 1968). Barcelona: Barcino.

Lafarga, Francisco y Pegenaute, Luis, eds. (2009): Diccionario histórico de la traducción en España. Madrid: Gredos.

LAfarga, Francisco (2004): Empuje cultural y freno económico: las traducciones literarias al castellano y al catalán en competencia. $V$ Colloque sur la traduction, la terminologie et l'interprétation. Consultado el 25 marzo 2013, <http://www.cttic.org/ACTI/2004/papers/ Lafarga_Habana.pdf $>$

LAfarga, Francisco (2006): Sobre la traducción en Cataluña en época reciente: cultura, comercio, competencia de lenguas. In: Consuelo Gonzalo García y Pollux Hernúñez, eds. Corcillvm. Estudios de traducción, lingüística y filología dedicados a Valentín García Yebra. Madrid: Arco/Libros, 569-576.

Llompart, Josep. M. (1966): «Pròleg del traductor», a Viatge al Pirineu de Lleida, de Camilo José Cela. . In: Montserrat Bacardí, Joan Fontcuberta y Francesc Parcerisas. Cent anys de traducció al català (1891-1990). Antologia, 189-192.

LóPEZ Del CASTILlo, Lluís (1976): Llengua estàndard i nivells de llenguatge. Barcelona: Laia.

López Estrada, Francisco (1993): El Tirante castellano de 1511 y los libros de viajes. Actes del Symposion Tirant lo Blanc. Barcelona: Quaderns Crema, 441-470.

Malblanc, Alfred (1961): Pour une stylistique comparée du français et de l'allemand. Paris: Didier.

Mallafrè, Joaquim (1991): Llengua de tribu i llengua de polis. Bases d'una traducció literaria. Barcelona: Quaderns Crema.

Marí, Anotni (1997): L'experiència de l'autotraducció. In: V Seminari sobre la Traducció a Catalunya. Barcelona: AELC, 53-63.

Martines, Vicent (1997): El Tirant poliglota. Barcelona: Curial-Publicacions de l'Abadia de Montserrat.

Martines, Vicent (1999): Sin adobo se podrán bien servir. Traducción y Filología: traducción al español de la lírica de Joan Roís de Corella. Revista de Filología Románica. 16:213-264.

Martines, Vicent (2000): La obra de Ausiàs March en el contexto de la lírica románica medieval. In: Enrique Nogueras y Lourdes Sánchez, eds. Ausiàs March y las literatura de su época. Granada: Universidad de Granada, 43-66.

Martines, Vicent (2005): Traducciones al servicio de la exégesis temprana sobre Ausiàs March y otros clásicos valencianos y el sentido de sus relaciones románicas. Revista de literatura medieval. 17:155-176.

Martines, Vicent (2006): Acaraments i contrastos literaris. Filologia i traducció per entendre Ausiàs March. Confronto letterario: Quaderni del Dipartimento di Lingue e Letterature Straniere Moderne de l'Università di Pavia. 46:235-268.

Martines, Vicent (2007): Materials per a l'anàlisi multilingüe i contrastiva de locucions, col-locacions i fraseologia. Un escandall quant a Ausiàs March i Tirant lo Blanch. Caplletra. Revista Internacional de Filologia. 40:153-191.

Martínez Romero, Tomás y Recio, Roxana, eds. (2001): Essays on Medieval Translation in the Iberian Península. Castelló-Omaha: Univ. Jaume I/Creighton Univ.

MéridA, Rafael M. (1993): ¿Las desgracias de un editor? Diego de Gumiel, Tirant el Blanc y Tirante el Blanco. In: Actas do IV Congresso Internacional da Associação de Literatura Hispánica Medieval. Lisboa: Edições

Morreale, Margherita (1958): Apuntes bibliográficos para la iniciación al estudio de las traducciones bíblicas medievales en catalán. Anacleta Sacra Tarraconensia, XXXI. Barcelona: Balmesiana.

PACTE (2000): Acquiring Translation Competence: Hypotheses and Methodological Problems in a Research Project. In: Alison Beeby, Doris Ensinger y Marisa Presas, eds. Investigating Translation. Amsterdam/ Filadelfia: John Benjamins, 99-106. 
PACTE (2001): La competencia traductora y su adquisición. Quaderns. Revista de traducció. 6:39-45.

Parra i Albà, Montserrat (1995): Adaptaciones teatrales de Los tres mosqueteros de Alejandro Dumas. In: Francisco Lafarga, Albert Ribas y Mercedes Tricàs, eds. La traducción. Metodología, historia, literatura: ámbito hispanofrancés. Barcelona: PPU, 255-261.

Payàs, Gertrudis (1999): Español de México y Español de España en la traducción de L'ombra de l'atzavara de Pere Calders. III Jornades de Traducció a Vic: Training Translators and Interpreters: New Directions for the Millenium. Vic, 12-15 mayo 1999.

Perarnau, Josep (1978): Aportació al tema de les traduccions bíbliques catalanes medievals. Revista Catalana de Teologia. 3:17-98.

Puig I TÀrrech, Armand (1987): Les traduccions bíbliques catalanes en el segle XIX. Revista Catalana de Teologia. XII (1):97-116.

Riera, CARMe (1997): L'autotraducció com a exercici de recreació. V Seminari sobre la Traducció a Catalunya. Barcelona: Associació d'Escriptors en Llengua Catalana, 45-53.

RierA I SANS, Jaume (1989): Catàleg d'obres en català traduïdes en castellà durant els segles XIV i XV. In: Antoni Ferrando, ed. Segon Congrés Internacional de la Llengua Catalana. Valencia: Institut de Filologia Valenciana-PAM, 699-709.

RiQuer, Martí de (1946): Traducciones castellanas de Ausiàs March en la Edad de Oro. Barcelona: Instituto Español de Estudios Mediterráneos.

RiQuer, Martí de (1974): Tirante el Blanco. Madrid: Espasa Calpe.

Riquer, Martí de (1990a): La difusió del Tirant lo Blanc. Aproximació al Tirant lo Blanc. Barcelona: Quaderns Crema, 241-248.

Riquer, Martí de (1990b): Ausiàs March, Poesías. (Traducido por Jorge De Montemayor). Barcelona: Planeta.

Riquer, Martí de (1991): Tirante el Blanco. Barcelona: Planeta.

RodríGUEZ-VIDA, Susana (1997): Catalán - Castellano frente a frente. Barcelona: Inforbook's S.L.

Romaguera, Joaquim (1988): Traduccions entre llengües de l'Estat español. Revista de Catalunya. 21:135-142.

Rullán, Ildefonso (1905): «Cuatre paraules d'es autor a n'es lectors», a Miquel de Cervantes Saavedra. L'enginyós hidalgo don Quixote de la Mancha. In: Montserrat BACARdí, Joan Fontcuberta y Francesc Parcerisas. Cent anys de traducció al català (1981-1990). Antologia. Vic: Eumo, 33-36.

Ruzicka Kenfel, Veljka y Lorenzo, Lourdes, eds. (2003): Estudios críticos de traducción de literatura infantil y juvenil. Análisis de las traducciones de obras inglesas y alemanas a las cuatro lenguas oficiales de España. Oviedo: Septem Ediciones.

SABAtÉ, Joan (1999): La publicitat en català. Barcelona: Pòrtic.

SÁnchez Rodrigo, Lourdes y Nogueras, Enrique (1996): Els poetes com a traductors: Kavafis en català, Kavafis en castellà. In: Miquel EDo, eds. Actes del Primer Congrés Internacional sobre Traducció. Barcelona: Publicacions de la Universitat Autònoma de Barcelona, 851-858.

SAntoyo, Julio César (1995): El siglo XIV: Traducciones y reflexiones sobre la traducción. In: Roxana Recio, ed. La traducción en España, ss. XIV-XVI. León: Universidad de León, 17-34.

SAntoyo, Julio César (1999): Traducciones cotidianas en la Edad Media: una parcela olvidada. In: Julio César Santoyo. Historia de la traducción: quince apuntes. León: Universidad de León.

SELLENT, Joan (1998): La traducció literària en català al segle XX: alguns títols representatius. Quaderns. Revista de Traducció. 2:23-32.

Tró, Josep (1982): L’ensenyament del català als no-catalanoparlants. $3^{\mathrm{a}}$ ed. Barcelona: Eumo.

Unversitat de BARCelona (1991): Documents administratius universitaris (DAU). Barcelona: Universitat de Barcelona, Servei de Llengua Catalana.

Universitat Jaume I (2003): Manual de documents i llenguatge administratiu. Castelló de la Plana: Publicacions de la Universitat Jaume I.

Universitat Pompeu Fabra (2004): Documents administratius de la Universitat Pompeu Fabra. Barcelona: Universitat Pompeu Fabra. 
Universitat de ValÈnCia (2007): Criteris lingüístics per als usos institucionals de les universitats valencianes. Consultado el 17 febrero 2007, <http://www.ua.es/spv/assessorament/ criteris.pdf>.

Vallverdú, Francesc (1998): Els problemes de la traducció. In: Montserrat Bacardí, Joan Fontcuberta y Francesc Parcerisas. Cent anys de traducció al català (1981-1990). Antologia. Vic: Eumo, 305-318.

VALOR, Enric (1979): Curs mitjà de gramàtica catalana referida especialment al País Valencià. $2^{\text {a }}$ ed. Valencia: 3 i 4.

VÁzQUez Ayora, Gerardo (1977): Introducción a la traductología. Washington DC: Georgetown University Press.

VIDAL JovÉ, Joan Francesc, ed. (1969): Tirante el Blanco. Madrid: Alianza.

Vinay, Jean-Paul y DarbeLnet, Jean (1958): Stylistique comparée du français et de l'anglais. Méthode de traduction. Paris: Didier.

Wittlin, Curt (1995): De la traducció literal a la creació literària. Valencia/Barcelona: Institut Interuniversitari de Filologia Valenciana/Publicacions de l'Abadia de Montserrat. 